\title{
Algebraic approach to vibrational spectra of tetrahedral molecules: a case study of silicon tetrafluoride
}

\author{
Xi-Wen Hou ${ }^{a, b}$, Shi-Hai Dong ${ }^{a}$, Mi Xie ${ }^{c}$, and Zhong-Qi Ma ${ }^{a}$ \\ ${ }^{a}$ Institute of High Energy Physics, P.O. Box 918(4), Beijing 100039, The People's Republic of China \\ ${ }^{b}$ Department of Physics, University of Three Gorges, Yichang 443000, The People's Republic of China \\ ${ }^{c}$ Department of Physics, Tianjin Normal University, Tianjin 3000\%, The People's Republic of China
}

\begin{abstract}
Both the stretch and bend vibrational spectrum and the intensity of infrared transitions in a tetrahedral molecule are studied in a $\mathrm{U}(2)$ algebraic model, where the spurious states in the model Hamiltonian and the wavefunctions are exactly removed. As an example, we apply the model to silicon tetrafluoride $\mathrm{SiF}_{4}$.
\end{abstract}

\section{INTRODUCTION}

In recent years, algebraic methods $[1,2]$ have been developed to be an effective theory for descriptions of vibrations, rotations, and rotation-vibration interactions in polyatomic molecules [3,4]. $\mathrm{U}(4)$ and $\mathrm{U}(2)$ algebraic model have mostly been used so far in the analysis of experimental data. U(4) model $[5,6]$ takes the rotation and the vibration into account simultaneously but becomes quite complex when the number of atoms in the molecules becomes larger than 4, while $\mathrm{U}(2)$ model is particularly well suited for dealing with the stretching vibrations of polyatomic molecules such as the octahedral and benzene-like systems $[7,8]$. Those two models are still extensively used for small molecules $[9,10]$. Recently, an active step in the development of algebraic models has been taken by Iachello and Oss to incorporate the bending modes into the models for benzene [11] and acetylene [12]. Extending $\mathrm{U}(2)$ algebraic model [3] to describe both the stretching and the bending vibrations in $\mathrm{X}_{3}[13]$ and $\mathrm{X}_{4}$ molecules [14], Frank and his co-workers have introduced a symmetry-adapted algebraic model, and established an explicit connection between algebraic and configuration space calculations. Developing the local mode model [15], we have recently proposed an algebraic model of boson realization [1618] for the complete vibrations by taking full advantages of the discrete symmetries of molecular systems. It is worth mentioning that $\mathrm{U}(\mathrm{k}-1)$ and $\mathrm{U}(\mathrm{n})$ algebraic approach have been introduced for the $\mathrm{k}=3 \mathrm{~m}-3$ rotational and vibrational degrees of freedom of $\mathrm{m}$-atomic molecules [19], and for $\mathrm{n}-1$ stretching vibrational degrees of freedom of polyatomic molecules [20], respectively. However, those two approaches are less feasible than $\mathrm{U}(4)$ and $\mathrm{U}(2)$ model in application.

In order to study vibrational modes of medium-size molecules, one usually chooses a tetrahedral molecule as one of good samples. Its excited stretching vibrational states were explained in the local mode model [21]. In our previous papers, the vibrational spectrum of methane was analyzed in terms of bosonic operators [16] and q-deformed harmonic oscillators [23], however its infrared intensity was not taken into account. Recently new algebraic SU(2) model [24] has been proposed for tetrahedral molecules. This model needs at least eight parameters for the calculation of transition intensities. The 
lack of experimental data did not allow such a fit for the methane molecule, and interactions between the stretch and the bend were neglected in the calculation of energy levels. Leroy et al. have up to date presented an algebraic model [22] only for highly excited stretching modes and infrared intensities in this system. Although a precise infrared transition model has been constructed in algebraic scheme, it will be complex when the bending vibrations are considered.

In this letter, both stretching and bending vibrations and intensities of infrared transition of all active modes in a tetrahedral molecule are studied in a $\mathrm{U}(2)$ algebraic model based on our new method for eliminating the spurious states and spurious components in Hamiltonian. Our model transition operator contains only five parameters. One of its applications is presented to silicon tetrafluoride $\mathrm{SiF}_{4}$. Calculated results demonstrate that our method for dealing with spurious states are also effective for $\mathrm{U}(2)$ algebraic model, which open the possibility to use this model for analyzing the complete vibrations in large molecules where spurious states exist. In Sec. II, considering the symmetry of a tetrahedral molecule, we set up the model Hamiltonian, in which the interactions related to spurious states are exactly removed. Its infrared intensity is studied in Sec. III. Application to $\mathrm{SiF}_{4}$ is given in Sec. IV, where symmetrized bases are used to simplify calculations. Concluding remarks are made in Sec. V.

\section{HAMILTONIAN}

For a tetrahedral molecule $\mathrm{XY}_{4}$, we introduce ten $\mathrm{U}(2)$ algebras to describe ten interactions between atoms: $\mathrm{U}_{j}(2)(1 \leq j \leq 4)$ for $\mathrm{X}-\mathrm{Y}$ and $\mathrm{U}_{\mu}(2)(5 \leq \mu \leq 10)$ for $\mathrm{Y}-\mathrm{Y}$ interactions. The assignments of the Cartesian coordinate system are the same as in Ref. [16]. Each $\mathrm{U}_{\alpha}(2)(1 \leq \alpha \leq 10)$ is generated by the operators $\left\{\hat{N}_{\alpha}, \hat{J}_{+, \alpha} \hat{J}_{-, \alpha}, \hat{J}_{0, \alpha}\right\}$, satisfying the following commutation relations:

$$
\begin{array}{ll}
{\left[\hat{J}_{0, \alpha}, \hat{J}_{ \pm, \beta}\right]= \pm \delta_{\alpha \beta} \hat{J}_{ \pm, \alpha},} & {\left[\hat{J}_{+, \alpha}, \hat{J}_{-, \beta}\right]=2 \delta_{\alpha \beta} \hat{J}_{0, \alpha},} \\
{\left[\hat{N}_{\alpha}, \hat{J}_{0, \beta}\right]=0,} & {\left[\hat{N}_{\alpha}, \hat{J}_{ \pm, \beta}\right]=0 .}
\end{array}
$$

where $\hat{N}_{\alpha}$ is related with the Casimir operator of $\mathrm{U}(2)$ :

$$
2 \hat{J}_{0, \alpha}^{2}+\hat{J}_{+, \alpha} \hat{J}_{-, \alpha}+\hat{J}_{-, \alpha} \hat{J}_{+, \alpha}=\hat{N}_{\alpha}\left(\hat{N}_{\alpha} / 2+1\right) .
$$

Denote by $v_{\alpha}$ the number of quanta in the $\alpha$ th bond. The local basis states for each bond are labeled by the eigenvalue $N_{\alpha}$ of $\hat{N}_{\alpha}$ and $v_{\alpha}$, and written as $\left|N_{\alpha}, v_{\alpha}\right\rangle$. Their products provide the local bases:

$$
\left|N_{1}, v_{1}\right\rangle\left|N_{2}, v_{2}\right\rangle \cdots\left|N_{10}, v_{10}\right\rangle \equiv\left|N_{\alpha}, v_{\alpha}\right\rangle
$$

Those $N_{j}$ of equivalent bonds are equal to each other: $N_{j}=N_{s}$, and $N_{\mu}=N_{b}$, where and hereafter, the indexes $j, \mu$, and $\alpha$ run from 1 to 4,5 to 10, and 1 to 10, respectively, and footnotes $s$ and $b$ refer to the $\mathrm{X}$-Y couplings and the Y-Y couplings, respectively.

There are three kinds of $\mathrm{O}(2)$ invariant combinations of those generators:

$$
\begin{aligned}
\hat{H}_{\alpha}^{M} & =\left(\hat{J}_{+, \alpha} \hat{J}_{-, \alpha}+\hat{J}_{-, \alpha}, \hat{J}_{+, \alpha}\right) / 2-\hat{N}_{\alpha} / 2, \\
\hat{H}_{\alpha, \beta} & =2 \hat{J}_{0, \alpha} \hat{J}_{0, \beta}-\hat{N}_{\alpha} \hat{N}_{\beta} / 2, \quad \alpha \neq \beta, \\
\hat{V}_{\alpha, \beta} & =\hat{J}_{+, \alpha} \hat{J}_{-, \beta}+\hat{J}_{-, \alpha} \hat{J}_{+, \beta}, \quad \alpha \neq \beta .
\end{aligned}
$$


Their matrix elements in the local bases are given in Ref. [13]. The operators $\hat{H}_{\alpha}^{M}$ correspond to the energy of the $\alpha$ th Morse oscillator. The operators $\hat{H}_{\alpha, \beta}$ describe anharmonic terms of the type $v_{\alpha} v_{\beta}$, while the operators $\hat{V}_{\alpha, \beta}$ describe interbond couplings which, in configuration space, are of the type $\mathbf{r}_{\alpha} \mathbf{r}_{\beta}$, where $\mathbf{r}_{\alpha}$ and $\mathbf{r}_{\beta}$ are the displacement vectors of bonds $\alpha$ and $\beta$ from their equilibrium positions.

Considering that $\sum \hat{J}_{+, \mu}$ (or $\sum \hat{J}_{-, \mu}$ ) relates only to spurious states [23], we obtain the following $T_{d}$ invariant Hamiltonian in terms of those three kinds of operators:

$$
\begin{aligned}
H= & H_{s}+H_{b}+H_{s b}, \\
H_{s}= & \lambda_{s 1} \sum_{j=1}^{4} \hat{H}_{j}^{M}+\lambda_{s 2} \sum_{i \neq j=1}^{4} \hat{H}_{i, j}+\lambda_{s 3} \sum_{i \neq j=1}^{4} \hat{V}_{i, j}, \\
H_{b}= & \lambda_{b 1} \sum_{\mu=5}^{10} H_{\mu}^{M}+\lambda_{b 2} \sum_{\nu-\mu \neq 3, \mu<\nu=6}^{70} \hat{H}_{\mu, \nu} \\
& +\lambda_{b 3} \sum_{\mu=5}^{7} \hat{H}_{\mu, \mu+3}+\lambda_{b 4} \sum_{\mu=5}^{7} \hat{V}_{\mu, \mu+3}, \\
H_{s b}= & \lambda_{s b 1}\left\{\sum_{\mu=5}^{7}\left(\hat{H}_{1, \mu}-\hat{H}_{1, \mu+3}\right)+\left(\hat{H}_{2,5}-\sum_{\mu=6}^{8} \hat{H}_{2, \mu}+\hat{H}_{2,9}+\hat{H}_{2,10}\right)\right. \\
& \left.+\sum_{\mu=3}^{5}\left(\hat{H}_{3,2 \mu}-\hat{H}_{3,2 \mu-1}\right)+\left(-\hat{H}_{4,5}-\hat{H}_{4,6}+\sum_{\mu=7}^{9} \hat{H}_{4, \mu}-\hat{H}_{4,10}\right)\right\} \\
& +\lambda_{s b 2}\left\{\sum_{\mu=5}^{7}\left(\hat{V}_{1, \mu}-\hat{V}_{1, \mu+3}\right)+\left(\hat{V}_{2,5}-\sum_{\mu=6}^{8} \hat{V}_{2, \mu}+\hat{V}_{2,9}+\hat{V}_{2,10}\right)\right. \\
& \left.+\sum_{\mu=3}^{5}\left(\hat{V}_{3,2 \mu}-\hat{V}_{3,2 \mu-1}\right)+\left(-\hat{V}_{4,5}-\hat{V}_{4,6}+\sum_{\mu=7}^{9} \hat{V}_{4, \mu}-\hat{V}_{4,10}\right)\right\},
\end{aligned}
$$

where $H_{s}, H_{b}$, and $H_{s b}$ describe the stretching interaction, the bending one, and the interaction between the stretching and bending modes, respectively. The Hamiltonian preserves the quantum number $V=\sum v_{\alpha}$.

\section{INTENSITIES OF INFRARED TRANSITION}

The infrared active mode is $F_{2}$. The absolute absorption intensities from state $v^{\prime}$ to $v$ are given by

$$
\begin{aligned}
& I_{v v^{\prime}}=\nu_{v v^{\prime}} P_{v v^{\prime}}, \\
& P_{v v^{\prime}}=\left|\left\langle v\left|\hat{T}_{x}\right| v^{\prime}\right\rangle\right|^{2}+\left|\left\langle v\left|\hat{T}_{y}\right| v^{\prime}\right\rangle\right|^{2}+\left|\left\langle v\left|\hat{T}_{z}\right| v^{\prime}\right\rangle\right|^{2},
\end{aligned}
$$

where $\nu_{v v^{\prime}}$ is the frequency of the observed transition, $\hat{T}_{x}, \hat{T}_{y}$, and $\hat{T}_{z}$ correspond to the three components of the infrared transition operator $\hat{T}$, and the state $|v\rangle$ denotes $\left|N_{\alpha}, v_{\alpha}\right\rangle$ for short. All other constants are absorbed in the normalization of the operator $\hat{T}$. The three components of $\hat{T}$ are

$$
\begin{aligned}
& \hat{T}_{x}=\zeta_{s}\left(\hat{t}_{1}-\hat{t}_{2}+\hat{t}_{3}-\hat{t}_{4}\right)+\zeta_{b}\left(\hat{t}_{6}-\hat{t}_{9}\right)+\zeta_{s b}\left(\hat{t}_{1}+\hat{t}_{2}+\hat{t}_{3}+\hat{t}_{4}\right)\left(\hat{t}_{6}-\hat{t}_{9}\right), \\
& \hat{T}_{y}=\zeta_{s}\left(\hat{t}_{1}-\hat{t}_{2}-\hat{t}_{3}+\hat{t}_{4}\right)+\zeta_{b}\left(\hat{t}_{7}-\hat{t}_{10}\right)+\zeta_{s b}\left(\hat{t}_{1}+\hat{t}_{2}+\hat{t}_{3}+\hat{t}_{4}\right)\left(\hat{t}_{7}-\hat{t}_{10}\right), \\
& \hat{T}_{z}=\zeta_{s}\left(\hat{t}_{1}+\hat{t}_{2}-\hat{t}_{3}-\hat{t}_{4}\right)+\zeta_{b}\left(\hat{t}_{5}-\hat{t}_{8}\right)+\zeta_{s b}\left(\hat{t}_{1}+\hat{t}_{2}+\hat{t}_{3}+\hat{t}_{4}\right)\left(\hat{t}_{5}-\hat{t}_{8}\right),
\end{aligned}
$$

where $\zeta_{s}, \zeta_{b}$, and $\zeta_{s b}$ are parameters, and $\hat{t}_{\alpha}$ is the local operator for the $\alpha$ th bond. The term with $\zeta_{s b}$ is one of the higher order contributions of $\hat{T}$, which is necessary for describing both the stretching and the bending active modes. The matrix elements of $\hat{t}_{\alpha}$ are taken to be [11] 


$$
\left\langle\hat{N}_{\alpha}, v_{\alpha}\left|\hat{t}_{\alpha}\right| \hat{N}_{\alpha}, v_{\alpha}^{\prime}\right\rangle=\exp \left(-\eta_{\alpha}\left|v_{\alpha}-v_{\alpha}^{\prime}\right|\right)
$$

Those $\eta_{j}$ for equivalent bonds are equal to each other: $\eta_{j} \equiv \eta_{s}$, and $\eta_{\mu} \equiv \eta_{b}$.

The five parameters in the transition operator of Eqs. (3.2)-(3.3) will be determined by fitting observed data. The calculated intensity can be used to check assignments and in the study of intramolecular energy relaxation in tetrahedral molecules.

\section{APPLICATION}

We now apply this model to study the complete vibrational spectrum and the infrared intensity of $\mathrm{SiF}_{4}$. To our knowledge, there are sixteen observed vibrational energy levels and thirteen infrared intensity data for $\mathrm{SiF}_{4}[25]$.

At first, we calculate the Hamiltonian matrix. The calculation for energy levels will be greatly simplified if the symmetrized bases are used. For the stretch and bend states of tetrahedral molecules, the symmetrized bases were given in Ref. [16], where the spurious states in the bases were eliminated. For stretching overtones of large molecules, the symmetrized bases have been recently presented by Chen et al. [26,27]. In those symmetrized bases the Hamiltonian matrix is a block one. According to Morse potentials for the stretch and the bend vibrations in the boson-realization model [28] for this molecule, we take two boson numbers $N_{s}$ and $N_{b}$ to be 100 and 15 , respectively. The parameters in Hamiltonian are determined by fitting the observed data, and given in $\mathrm{cm}^{-1}$ as follows:

$$
\begin{aligned}
& \lambda_{s 1}=-3.311, \quad \lambda_{s 2}=-4.331, \quad \lambda_{s 3}=-0.573, \quad \lambda_{b 1}=97.982, \quad \lambda_{b 2}=-1.197, \\
& \lambda_{b 3}=74.438, \quad \lambda_{b 4}=-4.173, \quad \lambda_{s b 1}=-0.145, \quad \lambda_{s b 2}=0.199 .
\end{aligned}
$$

The experimental data and the calculated values are listed in Table I. The standard deviation in this fit is $1.188 \mathrm{~cm}^{-1}$. From those parameters we can calculate the other values. It is worth mentioning that McDowell et al. [25] described the same energy levels by Dunham expansion with more parameters. This method does not provide explicitly wave functions so that some physical properties such as transition intensities are hard to be calculated.

$$
\text { Table I }
$$

Then, we compute the infrared intensity. Due to the Wigner-Eckart theorem, it is sufficient to calculate only the $z$ component of the transition operator, $\hat{T}_{z}$, in the symmetrized bases. Fitting the thirteen observed infrared intensities, we determine the parameters in the transition operator as follows:

$$
\eta_{s}=40.158, \quad \eta_{b}=22.853, \quad \zeta_{s}=3.614, \quad \zeta_{b}=3.286, \quad \zeta_{s b}=64.959 .
$$

In Table I we only list those calculated intensities to compare with known observed data. The other calculated intensities and energy levels can be obtained from us upon request.

One may observe in Table I that most of the calculated intensities are in good agreement with the experimental values, but a few are not. Those differences may come from that the observed intensities 
were only approximately accurate [25], and that the other higher order contributions to the operator

$\hat{T}$ were neglected. It is worth pointing out that there are also differences in magnitude between the calculated and the observed intensities for the stretching vibrations of octahedral molecules in $\mathrm{U}(2)$ model [29] because the same simple matrix elements Eqs.(3.3) were used. The more accurate experimental data are needed to improve the model.

\section{CONCLUDING REMARKS}

We have used a $\mathrm{U}(2)$ algebraic model for studying the stretching and bending vibrations and infrared intensity of a tetrahedral molecule. The model Hamiltonian with nine parameters and the model transition operator with five parameters provide quite good fits to the published experimental data of silicon tetrafluoride with standard deviations $1.188 \mathrm{~cm}^{-1}$ and 1.775 , respectively. This is based on our new methods for constructing symmetrized bases [16] and for removing both the spurious states in the wavefunction space and the spurious components in the Hamiltonian [23]. For comparison, we also studied this molecule in the boson-realization model, and obtained the corresponding standard deviations $1.985 \mathrm{~cm}^{-1}$ and 1.745 [28]. Through this example of applications of the model, we believe that our method for treating spurious states is useful for the model for other polyatomic molecules.

It is shown that anharmonic resonances, such as Darling-Dennison and Fermi resonances, are very important in descriptions of highly excited vibrational states in molecules. Darling-Dennison resonances can be included in the model by adding higher-order terms of the operators of Eq.(2.1). Fermi resonances can be taken into account using perturbation theory. It should be pointed out that Fermi resonances can be easily included in the extended local mode model [30,31] and the boson-realization model [17], while they are described by the nondiagonal matrix elements of Majorana operators in U(4) algebraic model. The coupling parameters in Hamiltonian we have obtained can be related to force field constants to be used in conjunction with a kinetic energy operator in a Schrödinger equation [32]. We will consider the physical meaning of the parameters in future investigations.

\section{ACKNOWLEDGMENTS}

The authors would like to thank Prof. Jin-Quan Chen and Dr. Jia-Lun Ping for useful discussions. This work was supported by the National Natural Science Foundation of China and Grant No. LWTZ1298 of the Chinese Academy of Sciences.

[1] F. Iachello, Chem. Phys. Lett. 78(1981), 581.

[2] F. Iachello and R. D. Levine, J. Chem. Phys. 77 (1982), 3046. 
[3] F. Iachello and R. D. Levine, Algebraic Theory of Molecules, Oxford Uni., Oxford, 1995.

[4] S. Oss, Adv. Chem. Phys., 93 (1996), 455.

[5] O. S. van Roosmalen, F. Iachello, R. D. Levine, and A. E. L. Dieperink, J. Chem. Phys. 79 (1983), 2515.

[6] O. S. van Roosmalen, I. Benjamin, and R. D. Levine, J. Chem. Phys. 81 (1984), 5986.

[7] F. Iachello and S. Oss, Phys. Rev. Lett. 66 (1991), 2976.

[8] J. L. Ping and J. Q. Chen, Ann. Phys. (N.Y.) 255 (1997), 75.

[9] T. Sako and K. Yamanouchi, Chem. Phys. Lett. 264 (1997), 403.

[10] I. L. Cooper and R. K. Gupta, Phys. Rev. A 55 (1997), 4112.

[11] F. Iachello and S. Oss, J. Chem. Phys. 99 (1993), 7337.

[12] F. Iachello and S. Oss, J. Chem. Phys. 104 (1996), 6956.

[13] A. Frank, R. Lemus, R. Bijker, F. Pérez-Bernal, and J. M. Arias, Ann. Phys. (N.Y.) 252 (1996), 211.

[14] F. Pérez-Bernal, R. Bijker, A. Frank, R. Lemus, and J. M. Arias, Chem. Phys. Lett. 258 (1996), 301.

[15] M. S. Child and L. Halonen, Adv. Chem. Phys. 57 (1984), 1.

[16] Z. Q. Ma, X. W. Hou, and M. Xie, Phys. Rev. A 53 (1996), 2173.

[17] X. W. Hou, M. Xie, and Z. Q. Ma, Phys. Rev. A, 55 (1997), 3401.

[18] X. W. Hou, M. Xie, and Z. Q. Ma, Inter. J. Theor. Phys. 36 (1997), 1153.

[19] R. Bijker, A. E. L. Dieperink, and A. Leviatan, Phys. Rev. A 52 (1995), 2786.

[20] C. Leroy and F. Michelot, J. Mol. Spectrosc. 151 (1992), 71.

[21] L. Halonen and M. S. Child, Mol. Phys. 46 (1982), 239.

[22] C. Leroy and V. Boujut,J. Mol. Spectrosc. 181 (1997), 127.

[23] M. Xie, X. W. Hou, and Z. Q. Ma, Chem. Phys. Lett. 262 (1996), 1.

[24] R. Lemus and A. Frank, J. Chem. Phys. 101 (1994), 8321.

[25] R. S. McDowell, M. J. Reisfeld, C. W. Patterson, B. J. Krohn, M. C. Vasquez, and G. A. Laguna, J. Chem. Phys. 77 (1982), 4337.

[26] J. Q. Chen, A. Klein, and J. L. Ping, J. Math. Phys. 37 (1996), 2400.

[27] J. Q. Chen and J. L. Ping, J. Math. Phys. 38 (1997), 387.

[28] X. W. Hou, M. Xie, S. H. Dong, and Z. Q. Ma, Ann. Phys. (N.Y.) (1998), in press.

[29] J. Q. Chen, F. Iachello, and J. L. Ping, J. Chem. Phys. 104 (1996), 815. 
[30] T. Lukka, E. Kauppi, and L. Halonen, J. Chem. Phys. 102 (1995), 5200.

[31] L. Halonen, J. Chem. Phys. 106 (1997), 7931.

[32] L. Halonen, J. Chem. Phys. 106 (1997), 831.

Table I. Observed and calculated energy levels and relative intensity

\begin{tabular}{ccc|ccc}
\hline \hline \multicolumn{3}{c|}{ Obs.[25] } & \multicolumn{4}{c}{ Calc. } \\
\hline$\Gamma$ & $E\left(\mathrm{~cm}^{-1}\right)$ & Intensity & $V$ & $E\left(\mathrm{~cm}^{-1}\right)$ & Intensity \\
\hline$E$ & 264.2 & & 1 & 264.415 & \\
$F_{2}$ & 388.4448 & 500 & 1 & 388.858 & 500.059 \\
$F_{2}$ & 776.3 & 0.9 & 2 & 775.327 & $2 \times 10^{-6}$ \\
$A_{1}$ & 800.8 & & 1 & 799.770 & \\
$F_{2}$ & 1031.3968 & 5000 & 1 & 1029.677 & 4999.867 \\
$E$ & 1064.2 & & 2 & 1064.186 & \\
$F_{2}$ & 1164.2 & 1.4 & 3 & 1164.169 & $3 \times 10^{-5}$ \\
$F_{2}$ & 1189.7 & 40 & 2 & 1188.631 & 40.065 \\
$F_{2}$ & 1294.05 & 2.4 & 2 & 1293.903 & $4 \times 10^{-4}$ \\
$F_{2}$ & 1418.75 & 0.1 & 2 & 1418.533 & 0.005 \\
$F_{2}$ & 1804.5 & 0.7 & 3 & 1804.706 & $5 \times 10^{-5}$ \\
$F_{2}$ & 1828.17 & 7 & 2 & 1828.745 & 3.888 \\
$F_{2}$ & 2059.1 & 1.2 & 2 & 2058.010 & 3.744 \\
$F_{2}$ & 2602.55 & 0.007 & 4 & 2603.789 & $4 \times 10^{-8}$ \\
$F_{2}$ & 2623.8 & 0.015 & 3 & 2623.678 & 0.001 \\
$F_{2}$ & 3068.5 & 0.015 & 3 & 3069.126 & $1 \times 10^{-5}$ \\
\hline \hline
\end{tabular}

